\title{
Research Paper \\ Psychometric Characteristics of Secondary Trauma Questionnaire (STQ) in Warfare
}

\author{
Yaser Rezapour Mirsaleh ${ }^{1},{ }^{*}$ Khodabakhsh Ahmadi $^{2}$
}

1. PhD of Counseling, Assistant Professor, Department of Counseling, Faculty of Psychology and Educational Sciences, Ardakan University, Ardakan, Iran. 2. PhD of Counseling, Professor, Behavioral Sciences Research Center, Institute of Neuroscience and Behavioral Sciences, Baqiyatallah University of Medical Sciences, Tehran, Iran.

\begin{tabular}{|c|c|}
\hline $\begin{array}{l}\text { Use your device to scan } \\
\text { and read the article online }\end{array}$ & \\
\hline & Citation: Rezapour Mirsaleh Y, Ahmadi Kh. [Psychometric Characteristics of Secondary Trauma Questionnaire (STQ) in \\
\hline 口Estring & Warfare (Persian)]. Iranian Journal of Psychiatry and Clinical Psychology. 2017; 23(3):348-361. https://doi.org/10.29252/NIRP. \\
\hline 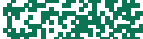 & IJPCP.23.3.348 \\
\hline 口ats & doil": https://doi.org/10.29252/NIRP.IJPCP.23.3.348 \\
\hline
\end{tabular}

Received: 13 Dec. 2015 Accepted: 17 Jan. 2017

Key words: Secondary Trauma Questionnaire, Warfare victims Validity, Reliability, Confirmatory factor analysis

\begin{abstract}
Objectives The purpose of the present study was to investigate some psychometric characteristics of Secondary Trauma Questionnaire (STQ) in the wives of warfare victims.

Methods Children of warfare victims living in Yazd, Esfahan, Shiraz, and Ahvaz and studying at Shahed and Isargar Schools of present in these cities in 2011-12 academic year were the target population for this study. Of this population, 379 subjects were selected by cluster sampling method. Secondary Trauma Questionnaire (STQ), Secondary Traumatic Stress Scale (STSS), Mississippi PTSD Scale, and Depression Anxiety Stress Scale (DASS) were used to collect the data. Confirmation factor analysis, Pearson correlation, and independent samples $\mathrm{t}$-test were used to analyze the data.

Results Findings of confirmation factor analysis revealed that STQ, similar to the one approved by the developers of this scale had a one-factor structure in Iranian samples. Face validity of STQ using item impact method and content validity of this scale using Content Validity Index (CVI) and Content Validity Ratio (CVR) were confirmed. There were significantly high correlations among the STQ scores of participants and STSS, PTSD Scale, and DASS that approved the convergent validity of the scale $(P<0.01)$. By using STQ, we were able to discriminate the group of subjects with high mental health from those with low mental health (discriminant validity). Conclusion The Farsi version of secondary trauma scale was valid and reliable for the wives of Iranian warfare victims.
\end{abstract}

\section{Extended Abstract}

\section{Introduction}

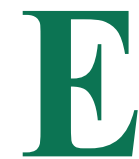

ffects of traumatic events of wars are not only limited to veterans but also affect their close relatives. Spouses of veterans are more exposed to physical violence, and emotional and verbal abuses [2]. Psychological distress experienced by spouses of veterans results from life with veterans who themselves are victims of war trauma, known as secondary traumatic stress [3]. Secondary traumatic stress is a syndrome of Post-Traumatic Stress Disorder (PTSD), which results from spending life with a person who has experienced some kind of psychological trauma [10].

Studies about secondary traumatic stress were seriously carried out in the 1990s, until then limited studies had been carried out about secondary trauma [10] because only limited scales were designed to measure secondary trauma [11]. Before specialized scales were designed to measure the symptoms of secondary traumatic stress, PTSD symptom

* Corresponding Author: Khodabakhsh Ahmadi, PhD

Address: Behavioral Sciences Research Center, Institute of Neuroscience and Behavioral Sciences, Baqiyatallah University of Medical Sciences, Tehran, Iran Tel: +98 (21) 88053767

E-mail:kh_ahmady@yahoo.com 
scales were often used to measure it. But, researchers have suggested that a special scale is needed to evaluate the special symptoms exhibited by people with secondary traumatic stress disorders, which is very different from PTSD [18]. Iran has experienced a prolonged war that lasted for eight years and therefore has many war veterans. Therefore, a scale to evaluate the secondary traumatic stress symptoms of spouses of veterans is necessary.

One of the most prevalent scales used to measure symptoms of secondary traumatic stress and used in many studies is Secondary Trauma Questionnaire (STQ). STQ was designed by Motta, Joseph, Rose, Suozzi, Leiderman [12] based on PTSD symptoms in DSM-IV and Figley's Compassion Fatigue Self-Stress Test [10]. The purpose of present study was to investigate some psychometric characteristics of STQ in wives of warfare victims.

\section{Methods}

Target population of this study included spouses of warfare victims living in Yazd, Esfahan, Shiraz, and Ahvaz and whose one of the children studied at Shahed and Isargar schools (special school for children of warfare victims in Iran) present in these cities, in the 2012-2013 academic year. Sample selection between veterans who their children studies in special schools for veterans caused by better availability to target population and sampling in schools. Criterion for being a war veteran victim was physical or psychological injuries caused by war accidents, which was documented in the academic records of the students. Spouses of veterans who were selected for inclusion in the target population of the study included women who married men injured in war, were aware of injuries caused to their future husbands at the time of marriage, and those who shared life with their husbands at the time of the study. Of this population, 379 subjects were selected by cluster sampling method. STQ, Secondary Traumatic Stress Scale (STSS), Mississippi PTSD Scale, and Depression Anxiety Stress Scale (DASS) were used to collect the data. First, STQ was carefully translated and then the translations were evaluated for their correctness by two English literature experts. The Translated items were again translated back to English by a specialist to confirm the Persian translation. Confirmation factor analysis, Pearson correlation, and independent samples t-test were used to analyze the obtained data.

\section{Results}

Face validity of STQ using item impact method and content validity of this scale using Content Validity Index (CVI) and Content Validity Ratio (CVR) were confirmed. Face validity of STQ was used to evaluate 22 spouses. They were asked to indicate the importance of the items on 5 Likert range from 1 (not important) to 5 (very important). Impact item method was used to calculate face validity of the items [29]. The results show that all scores were above 1.5 (Acceptable minimum score was 1.5).

Twelve faculty members specializing in PTSD were asked to assess content validity. They confirm items about difficulty level, ambiguity (flute interpret about items and incorrect meaning), Persian grammar, use appropriate words and item

Table 1. Regression weights and model fitness indices

\begin{tabular}{|c|c|c|c|c|c|}
\hline Item Number & $\begin{array}{c}\text { Standard Regression } \\
\text { Weights }\end{array}$ & Item Number & $\begin{array}{c}\text { Standard Regression } \\
\text { Weights }\end{array}$ & $\begin{array}{l}\text { Model Fitness } \\
\text { Indices }\end{array}$ & C.R \\
\hline 1 & $0.252^{*}$ & 13 & $0.536^{*}$ & $\chi^{2}$ & $222.351^{*}$ \\
\hline 2 & $0.321^{*}$ & 14 & $0.666^{*}$ & $\mathrm{FD} / \chi^{2}$ & 1.823 \\
\hline 3 & $0.641^{*}$ & 15 & $0.588^{*}$ & GFI & 0.939 \\
\hline 4 & $0.647^{*}$ & 16 & $0.673^{*}$ & AGFI & 0.914 \\
\hline 5 & $0.714^{*}$ & 17 & $0.668^{*}$ & RMR & 0.059 \\
\hline 6 & $0.777^{*}$ & 18 & $0.505^{*}$ & NFI & 0.934 \\
\hline 7 & $0.606^{*}$ & & & RFI & 0.917 \\
\hline 8 & $0.722^{*}$ & & & IFI & 0.969 \\
\hline 9 & $0.785^{*}$ & & & TLI & 0.961 \\
\hline 10 & $0.790^{*}$ & & & CFI & 0.969 \\
\hline 11 & $0.810^{*}$ & & & PRATIO & 0.797 \\
\hline 12 & $0.510^{*}$ & & & RMSEA & 0.048 \\
\hline
\end{tabular}


Table 2. Correlations of STQ scores with STSS, PTSD and DASS scores

\begin{tabular}{|c|c|c|c|c|c|c|c|c|c|c|}
\hline$\frac{y}{\frac{0}{0}}$ & $\stackrel{\check{n}}{n}$ & 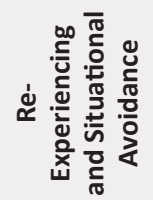 & 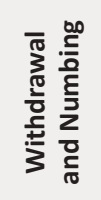 & 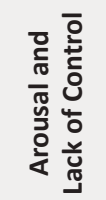 & 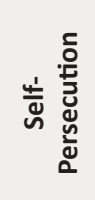 & 옴 & $\begin{array}{l}\frac{\vec{u}}{x} \\
\frac{\vec{x}}{\alpha}\end{array}$ & 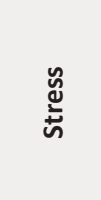 & $\begin{array}{l}\frac{.}{y} \\
\grave{y} \\
\frac{0}{0}\end{array}$ & 气̆ \\
\hline STQ & $0.853^{*}$ & $0.634^{*}$ & $0.409^{*}$ & $0.641^{*}$ & $0.458^{*}$ & $0.678^{*}$ & $0.696^{*}$ & $0.711^{*}$ & $0.703^{*}$ & $0.745^{*}$ \\
\hline
\end{tabular}

Table 3. Comparison of STQ scores in high and low mental health groups (discriminant validity)

\begin{tabular}{|c|c|c|c|c|c|c|c|}
\hline Variables & Groups & Number & Mean & SD & T Score & df & Sig. \\
\hline \multirow{2}{*}{$\begin{array}{c}\text { Secondary } \\
\text { trauma }\end{array}$} & High mental health & 50 & 22.32 & 4.01 & \multirow{2}{*}{-16.887} & \multirow{2}{*}{98} & \multirow{2}{*}{0.0001} \\
\hline & Low mental health & 50 & 57.28 & 14.08 & & & \\
\hline
\end{tabular}

allocation. Waltz \& Bausell method [30] was used to assess Content Validity Index (CVI). Experts evaluate "Relevance", "Clarity" and "Simplicity" of items on 4-point Likert scale. The results show that all scores were above the acceptable criteria (regarding number of experts) 0.79 [31-33]. The scores ranged from 0.84 to 1 for relevance, clarity, and simplicity. Finally, CVR was calculated by Lawshe formula [34]. The results show that the scores were above acceptable criteria of 0.49 .

In the present study, confirmatory factor analysis was used to evaluate factorial structure of STQ. Findings of the confirmation factor analysis revealed that STQ, which is similar to the one approved by the developers of this scale has a one-factor structure in Iranian samples. Model fitness indices are shown in Table 1. Correlation of STQ scores with STSS, PTSD, and DASS scores was calculated to assess convergent validity of STQ (Table 2). All correlations are high and significant $(\mathrm{P}<0.01)$, showing that STQ has appropriate convergent validity with STSS, DASS, and PTSD scales.

To evaluate the discriminant validity of STQ, and DASS scores considered as mental health criteria we hypothesized individuals having high scores in DASS (have low mental health) than who have low scores in DASS, and also have high scores in STQ. First, the discriminant validity of individuals is evaluated and then arranged from bottom to top based on DASS scores. Fifty subjects who had high scores in DASS and another 50 who had low scores in DASS were considered as low and high mental health groups respectively. STQ can help in discriminating high mental health groups from low mental health groups (Table 3).

Cronbach alpha for total items was 0.92 . Test-retest reliability of STQ was assessed by administrating the scale twice at an interval of one week. Correlation between the two scores was $0.81(\mathrm{P}<0.01)$. This result shows that Farsi version of STQ has appropriate reliability quotient.

\section{Conclusion}

Results show that Farsi version of the secondary trauma scale is valid and reliable for wives of Iranian warfare victims. Therefore, instead of using PTSD scales to evaluate the symptoms of secondary trauma in the wives of veterans, STQ can be used as a special scale. However, due to the limitations of the present research, it is necessary to take precautions while generalizing the results. One of the limitations of the present study was that veterans with PTSD were not separated from veterans without PTSD because of the limitations of the researcher and the need for a high sample for standardization. Limitation in sampling such as including only those spouses in the study who had married their husbands after they were victimized, or spouses of veterans who had a child in Shaheh and Isargar schools (not including spouses of all veterans), and sampling only from four cities of Iran, generalization of the results of the study was limited to all Iranian veteran spouses.

\section{Compliance With Ethical Guideline, Funding, Conflict of Interest, Acknowledgements}

In the present study, some items were considered as: awareness of the participants about the goals of the research, their satisfaction to participate in the research, and assuring the participants about the confidentiality of their personal information. This research did not receive any specific grant from funding agencies in the public, commercial, or not-for-profit sectors. The authors declare no conflicts of interest. We thank spouses of all the veterans who participated in the study. We are also immensely grateful to the school administrators for selecting the participants. 


\title{
ويثَّى هاى روانسنجى يرسشنامه آسيب ثانويه در همسران جانبازان
}

\author{
ياسر رضايور ميرصالح'، “خدابخش احمدى'
}

1- دكثراي مشاوره، استاديار، كروه راهنمايى و مشاوره، دانشكده روانشناسى و علوم تربيتى، دانشعاه اردكان، اردكان، ايران.

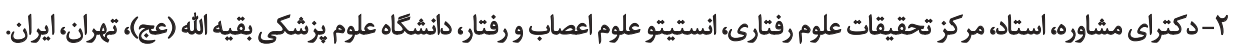

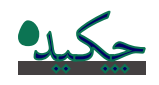

هلهدأ يُروهش حاضر با هدف بررسى برخى الز ويرّكى هانى روائسنجى يرسشئامه آسيب ثانويه در همسران جانبازان أنجام شد.

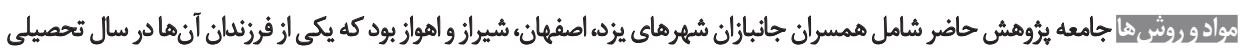

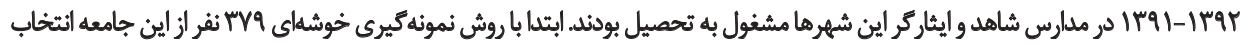

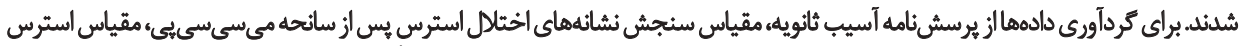

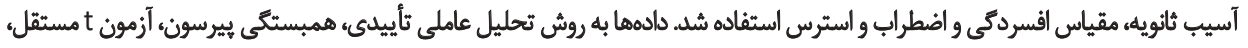

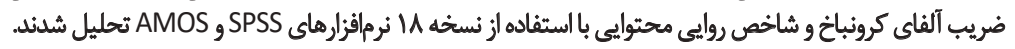

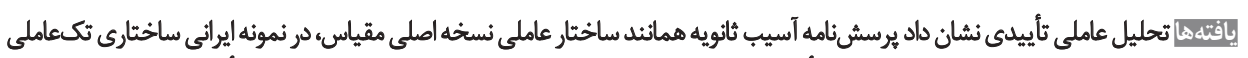

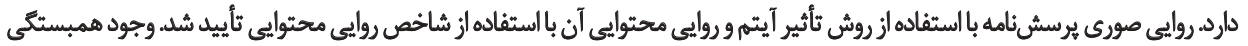

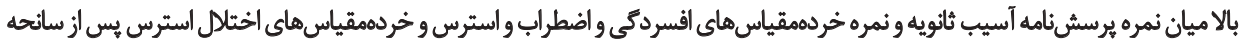

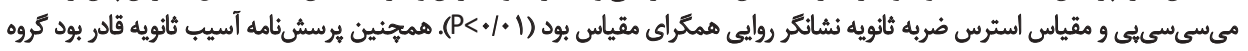

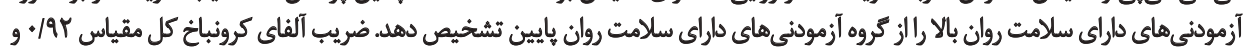

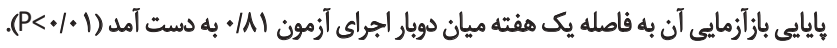

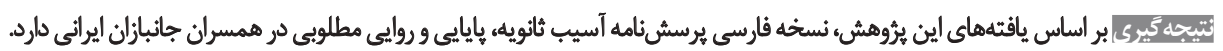

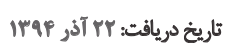

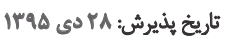

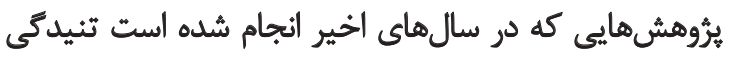

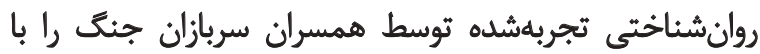

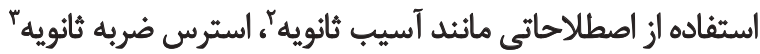

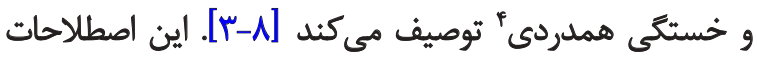

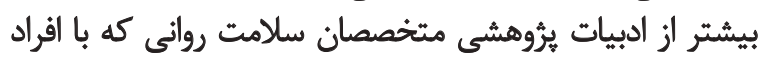

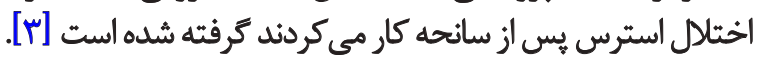

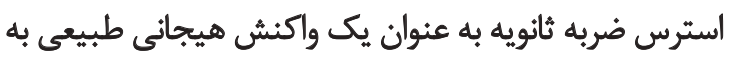

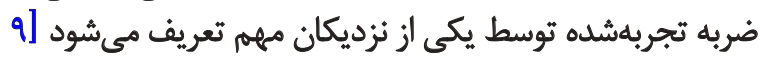

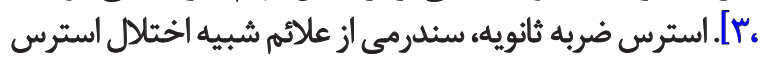

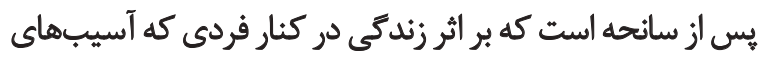

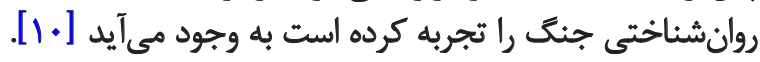

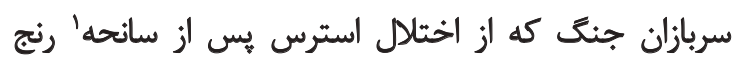

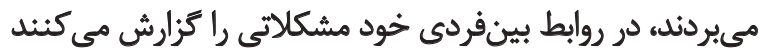

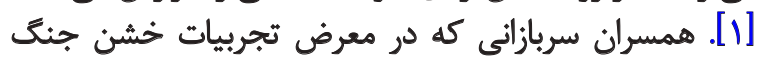

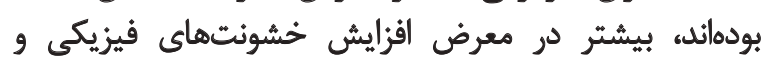

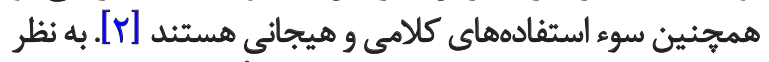

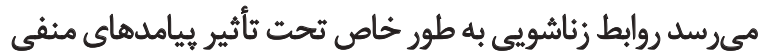

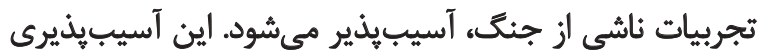

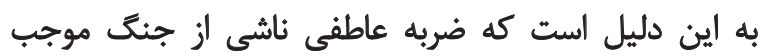

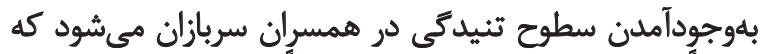

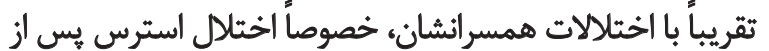
سانحه موازى است [r]

2. Secondary dramatization

3. Secondary trauma stress

4. Compassion fatigue

1. Posttraumatic Stress Disorder (PTSD)

$$
\text { ㄷ......... }
$$

: نويسئده مسئول:

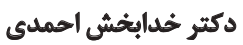

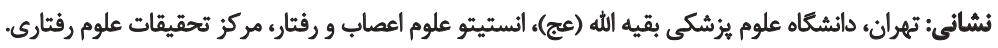

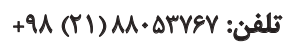

يست الكترونيكي: kh_ahmady@yahoo.com 
خصوصاً در سال هاى اوليه ازدواج كه اساس شكل گيرى احساسات زن و شوهر نسبت به يكديكر است، منجر شود مشكلات شوهر ت تأثير كمترى بر زن بكذارد.

ملاك جانبازبودن همسر، صدمه جسماني يا روانشناختي

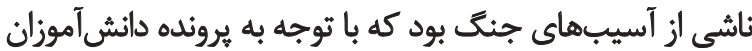

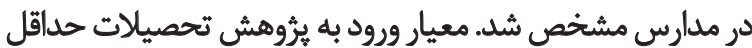

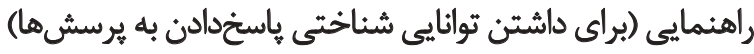

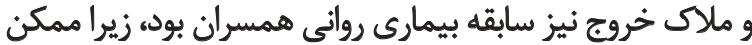

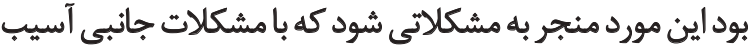

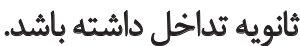

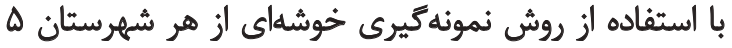

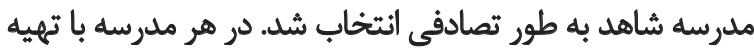

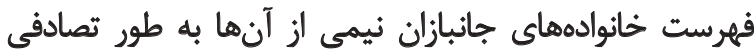

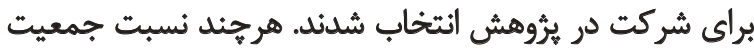

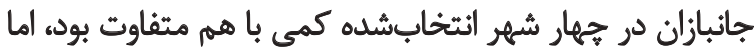

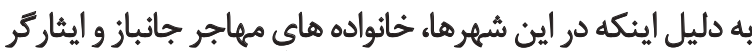

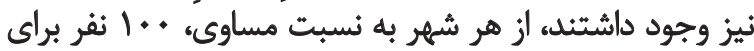

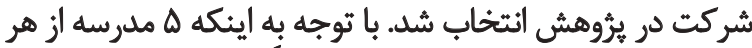

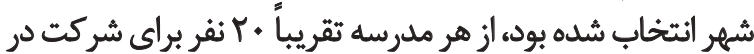

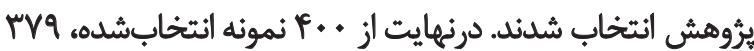

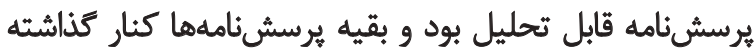

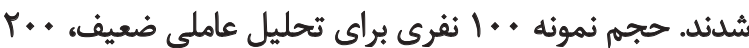

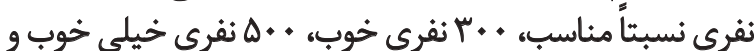

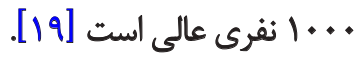

با جستوجو در منابع مختلف يُروهشى و با استفاده از إزئ

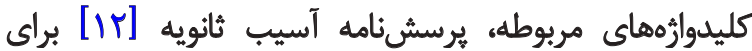

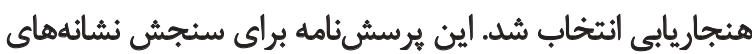

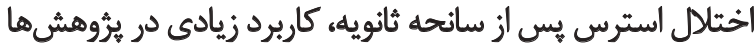

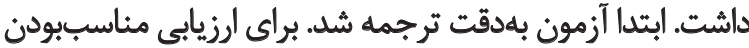

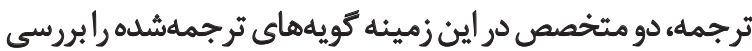

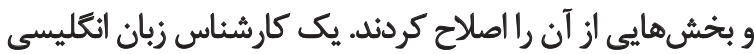

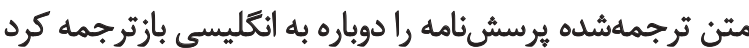

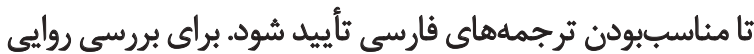

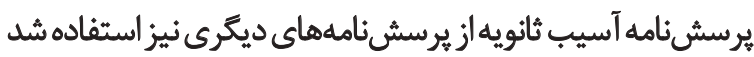

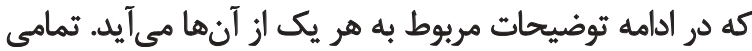

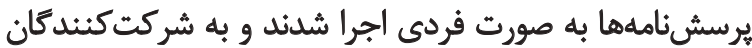

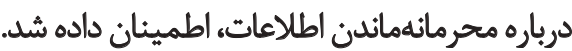

$$
\text { برسش نامه آسيب ثائوية }
$$

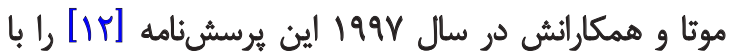

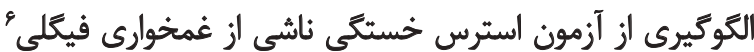

6. Figley's compassion fatigue self stress test
بنابراين همسران داراى استرس ضربه نيابى به عنوان افرادى إنى

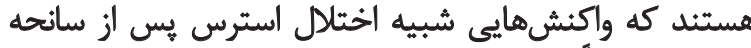

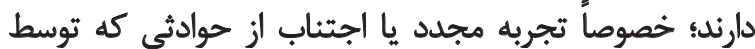

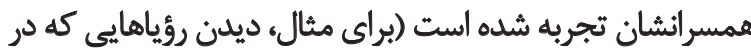

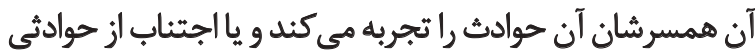

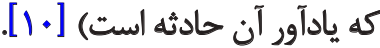
مطالعه و يثوهش در زمينه استرس ضربه ثانويه در اواخر دها

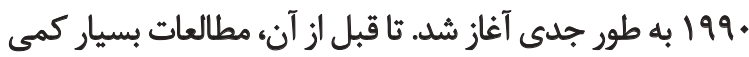

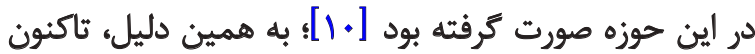

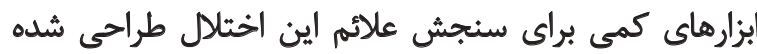

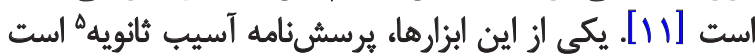

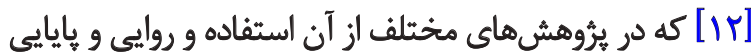

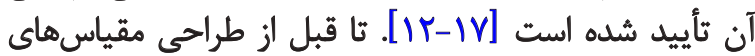

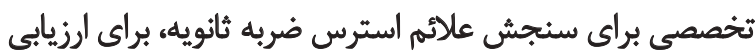

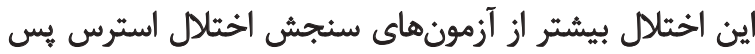

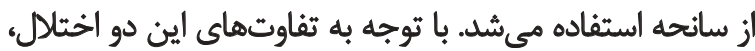

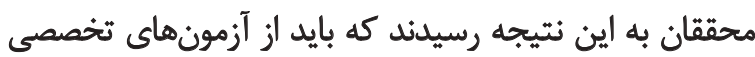

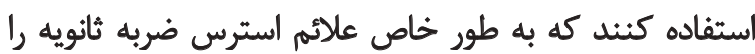
بررسى مى كنيند [1/] با توجه به اينكه كشور ما هشت سال جنكَ تحميلى را يشت

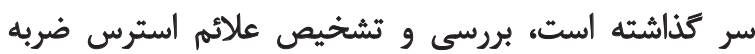

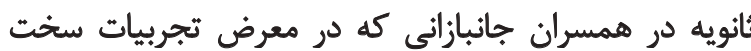

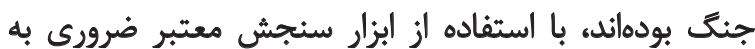

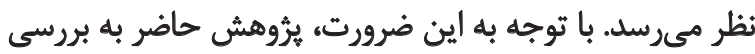

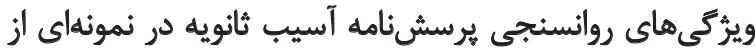
همسران جانبازان جنى تحميلى مى يردازد.

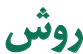

تحقيق حاضر يُروهشى توصيفى از نوع همبستكى است كه

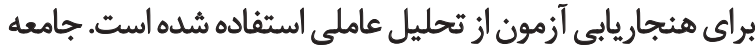

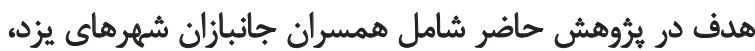

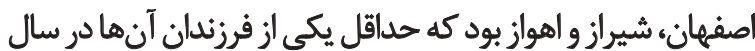

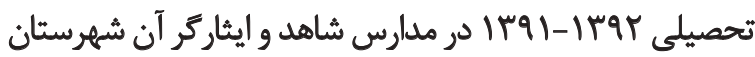

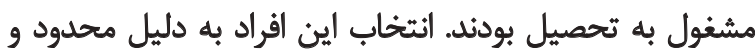

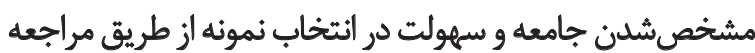

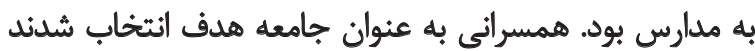

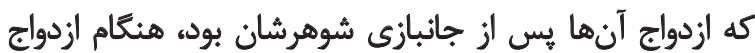

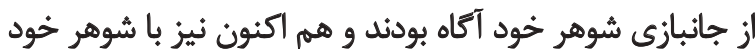

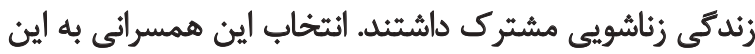

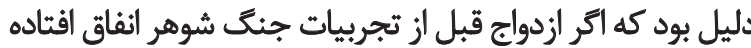

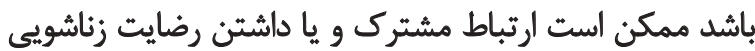

5. Secondary Trauma Questionnaire (STQ) 
از همسران جانبازان ايرانى [rr] و نمونداى از فرزئدان جانبازان [YF]

مقياس افسردتّى و اضطراب و استرس (I)-DASS)

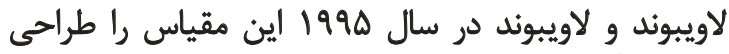

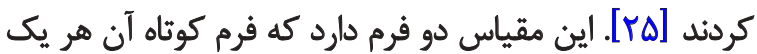

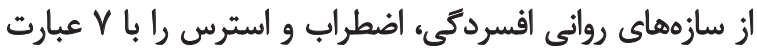

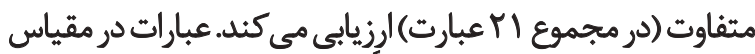

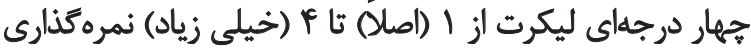

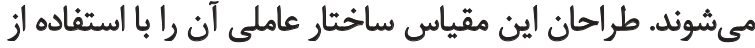

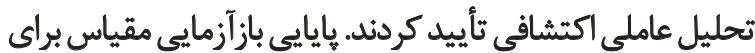

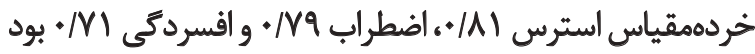

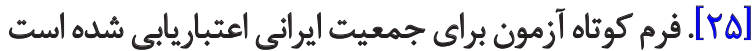

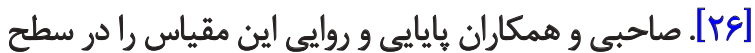

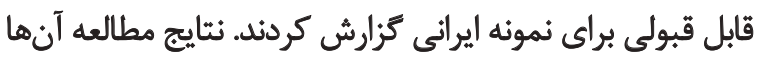

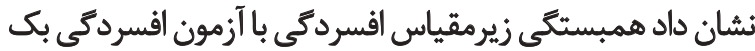

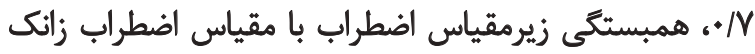

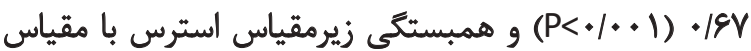

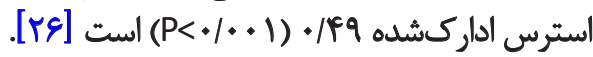

مقياس سنجش علائم اخثتلال استرس يس از سانحه ميسيسيني

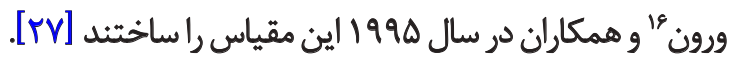

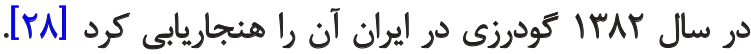

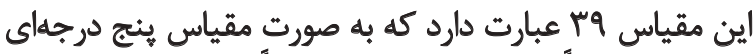

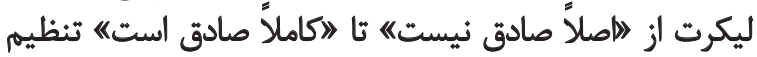

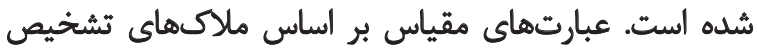

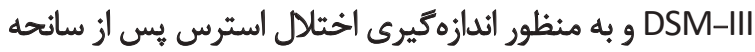

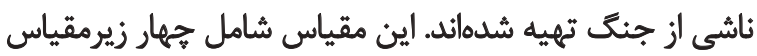

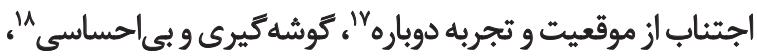

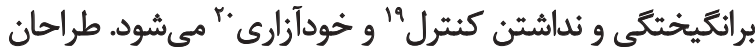

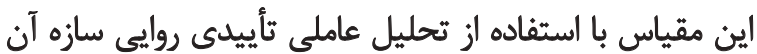

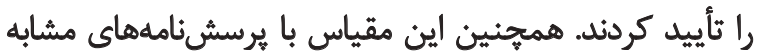

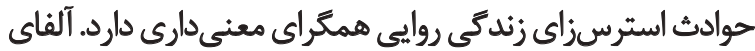

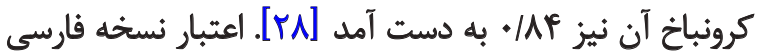

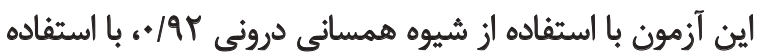

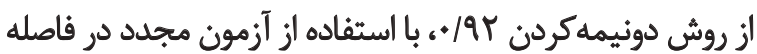

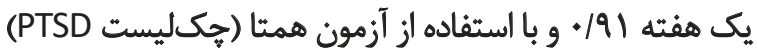

16. Vreven

17. Re-experiencing and situational avoidance

18. Withdrawal and numbing

19. Arousal and lack of control

20. Self persecution

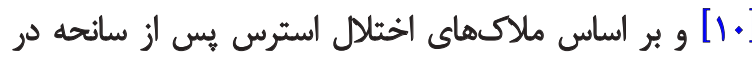

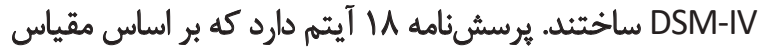

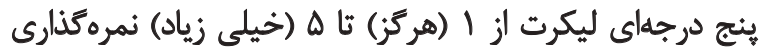

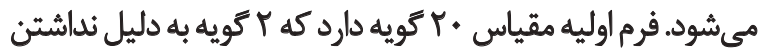

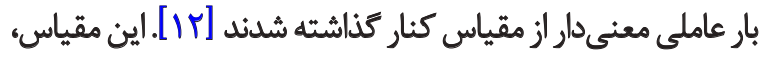

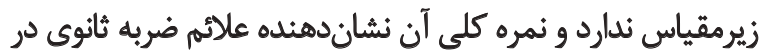

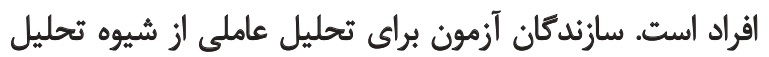

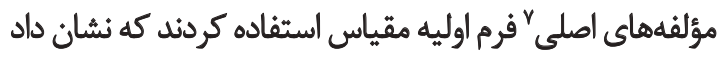

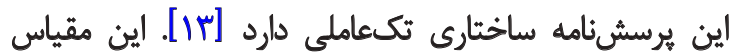

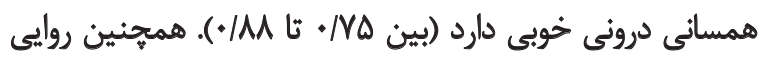

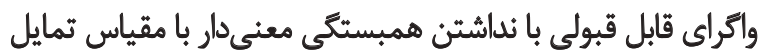

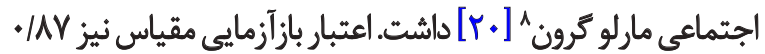

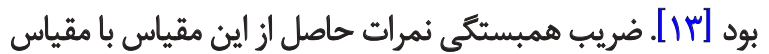

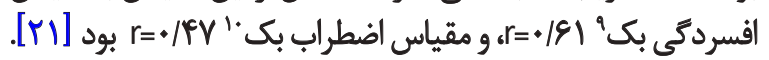

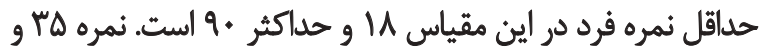

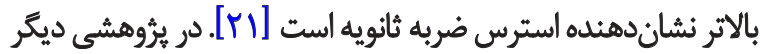

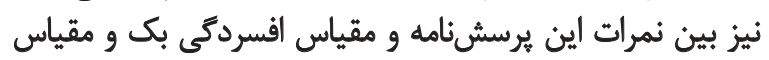

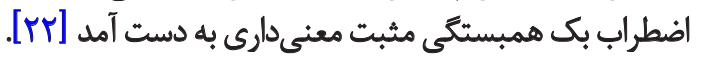

$$
\text { مقياس استرس ضربله ثائويه" }
$$

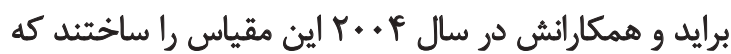

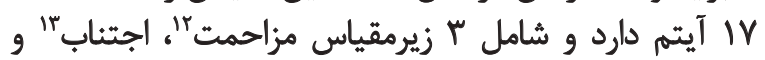

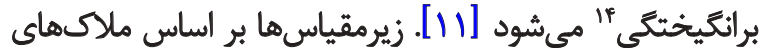

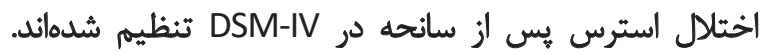

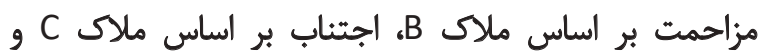

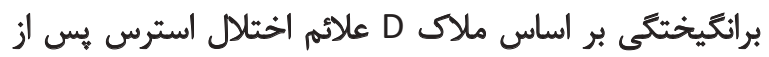

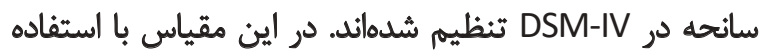

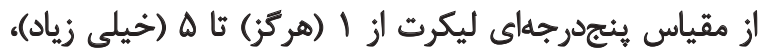

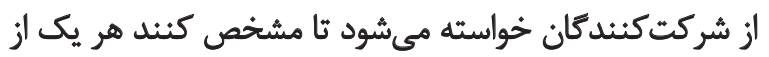

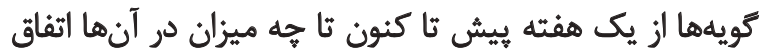

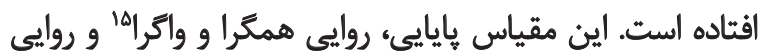

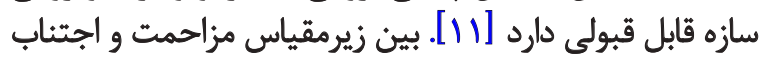

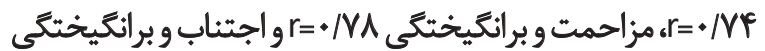

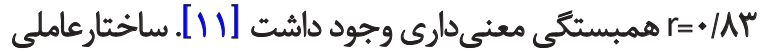

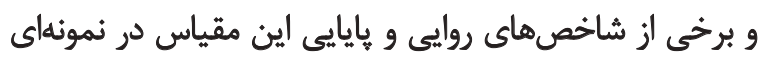

7. Principal components analysis

8. Marlowe Crowne social desirability scale

9. Beck Depression Inventory (BDI)

10. Beck Anxiety Inventory (BAI)

11. Secondary Traumatic Stress Scale (STSS)

12. Intrusion

13. Avoidance

14. Arousal

15. Discriminant validity 
هجهار قسمتى مشخص كر دند. نتايج فرمول محاسبه شاخص روايى باني

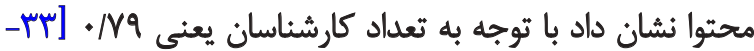

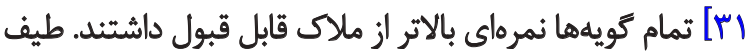

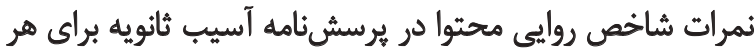

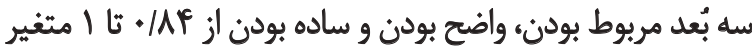

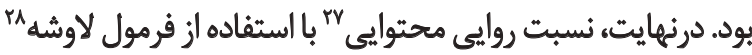

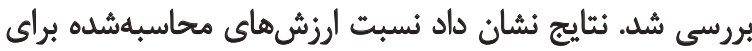

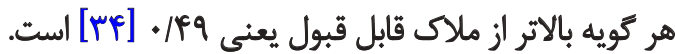

$$
\text { روايي سازه }
$$

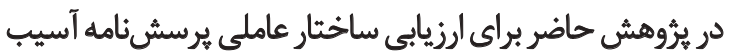

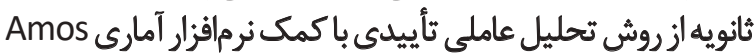

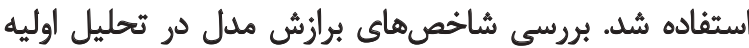

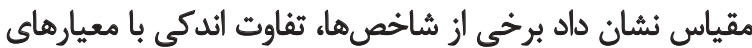

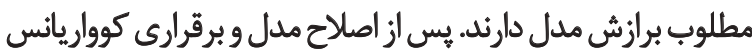

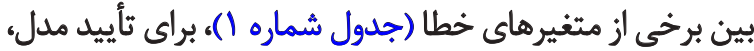

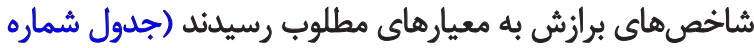

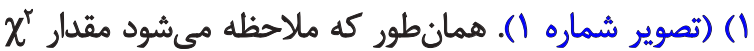

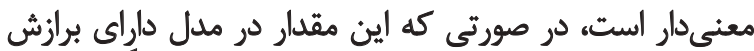

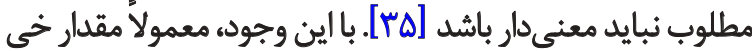

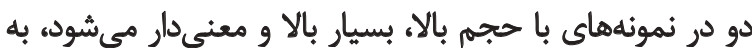

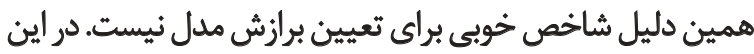

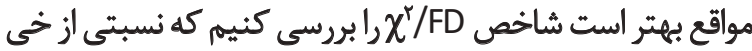

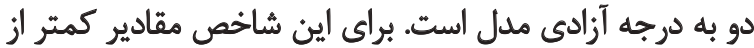

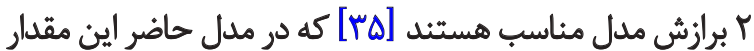

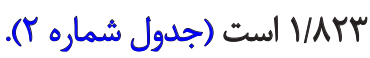

شاخصهاى نيكويى برازش"ج شاخص برازش هنجارشده"،

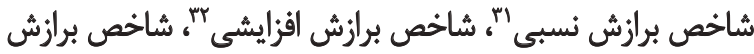

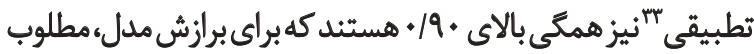

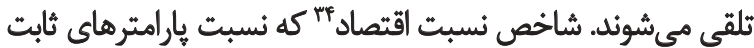

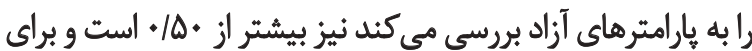

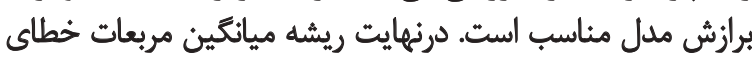

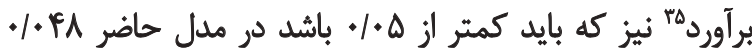

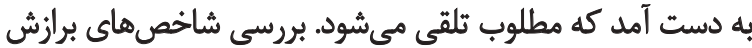

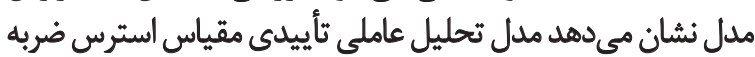

27. Content validity ratio

28. Lawshe

29. Goodness of Fit Index (GFI)

30. Normal Fit Index (NFI)

31. Relative Fit Index (RFI)

32. Incremental Fit Index (IFI)

33. Comparative Fit Index (CFI)

34. Parsimony Ratio (PRATIO)

35. Root Mean Square Error of Approximation (RMSEA)

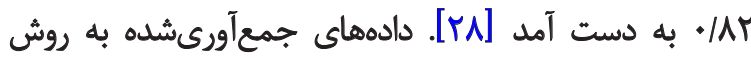

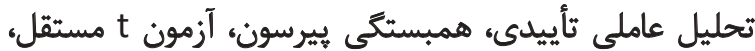

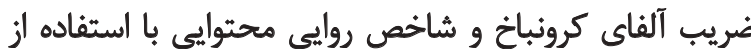

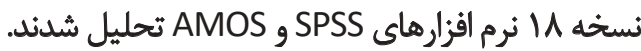

كافتهنها

بررسى اطلاعات جمعيت شناختى آزمودنى هاى شركت كنينده

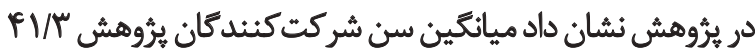

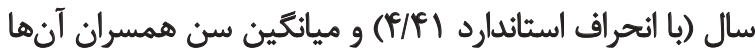

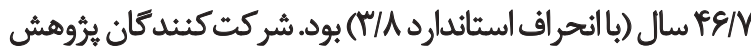

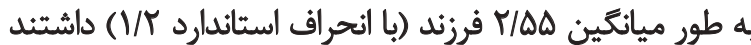

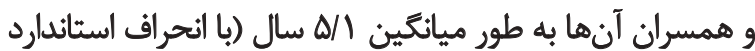

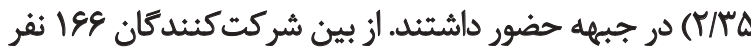

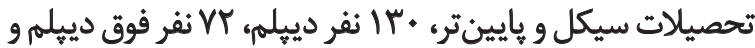

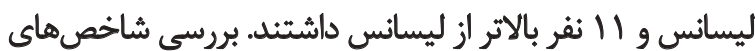

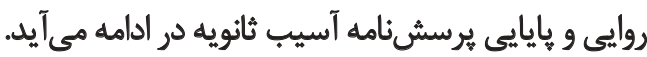

$$
\text { روائي صورى و محتوايى }
$$

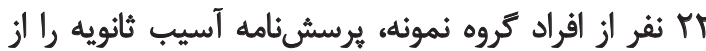

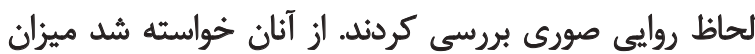

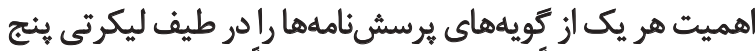

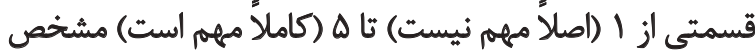

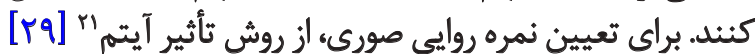

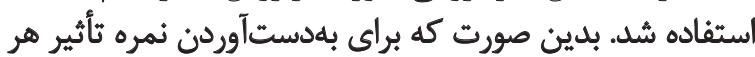

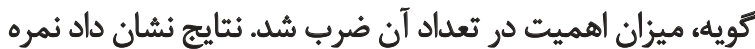

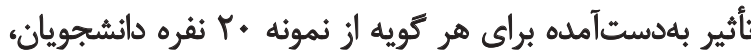

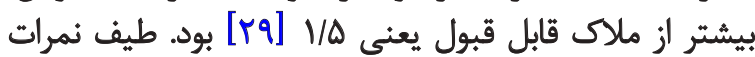

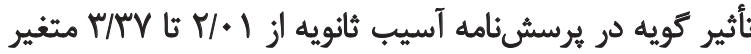

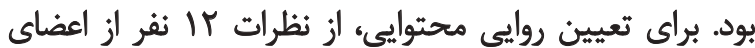

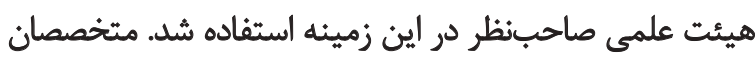

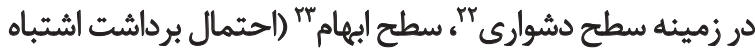

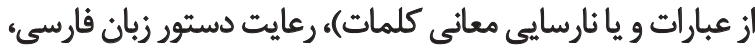

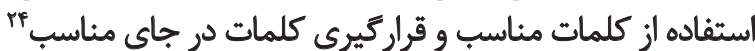

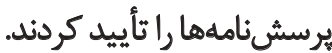

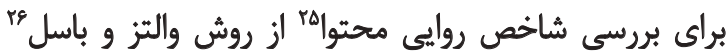

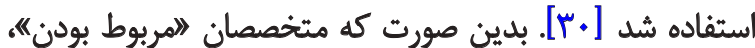

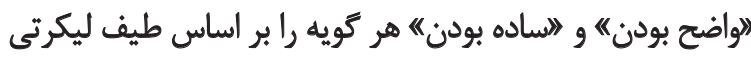

21. Item impact

22. Difficulty

23. Ambiguous

24. Item allocation

25. Content validity index

26. Waltz and Bausell 
جدول ا.وزنهاى ركرسيونى كويهها يِ از اصلاح مدل و شاخصهاى برازش مدل

\begin{tabular}{|c|c|c|c|c|c|}
\hline C.R & شاخصهاي برازش مدل & وزن رتاندرسيونى & شماره كويه & وزنانداردشيدهى & شوماره \\
\hline MTY/TQI* & $\chi^{Y}$ & . & ir & . /TQT" & 1 \\
\hline V/ATr & $\chi^{\Upsilon} / F D$ & $.1999^{\circ}$ & if & ./MTI" & $r$ \\
\hline . & GFI &.$/ \Delta M^{\circ}$ & 10 &.$|99|^{*}$ & $r$ \\
\hline.$/ 919$ & AGFI & $.18 n$ & 18 & . Iapr & $\varphi$ \\
\hline .1 .09 & RMR & $199 \Lambda^{\circ}$ & IV & .MIE" & $\Delta$ \\
\hline.$/ 947$ & NFI & $\cdot / \Delta \cdot \Delta^{\circ}$ & M & $\cdot / m r^{*}$ & 8 \\
\hline.$/ 91 \mathrm{~V}$ & RFI & & & $.19 .9^{\circ}$ & $\checkmark$ \\
\hline.$/ Q 99$ & IFI & & & .Mrr" & $\wedge$ \\
\hline.$/ 981$ & TLI & & &.$/ N A \Delta^{\circ}$ & 9 \\
\hline.$/ 959$ & $\mathrm{CFI}$ & & &.$/ v a *^{*}$ & 1. \\
\hline . NaY & PRATIO & & &.$\left.|A|\right|^{*}$ & 11 \\
\hline 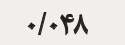 & RMSEA & & & $.|\Delta|+^{*}$ & ir \\
\hline
\end{tabular}

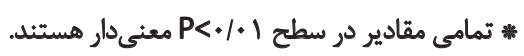

جدول r. مقايسه نمرات برسشنامه آسيب ثانويه در آزمودنىهاى با سلامت روان بالاو وايين (روايى افتراقى)

\begin{tabular}{|c|c|c|c|c|c|c|c|}
\hline معنى دطارى & آرادج & نمره T & انحراف استاندارد & مياتعين & تعداد & كروهها & متغيرها \\
\hline $.1 \ldots+1$ & $u$ & $-I E / A Y$ & $\begin{array}{l}\varphi / \cdot 1 \\
1 \% / \cdot 1\end{array}$ & $\begin{array}{l}T / M T \\
\Delta V / T A\end{array}$ & هـ هـ نهر. & سلامت بالاهت & آسيب ثانويه \\
\hline
\end{tabular}

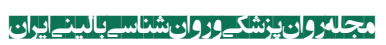

مقياس افسردكى و اضطراب و استرس از كم به زياد مرتب شديند.

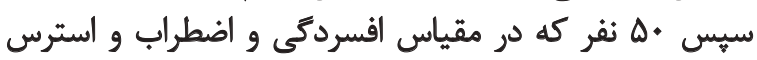

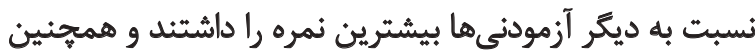

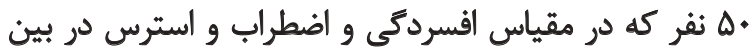

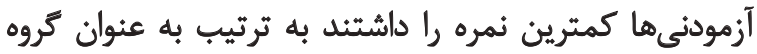

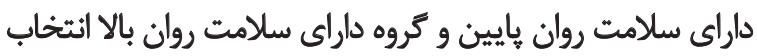

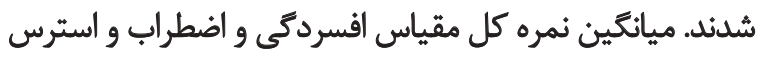

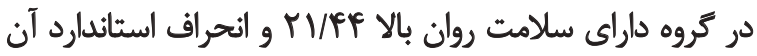

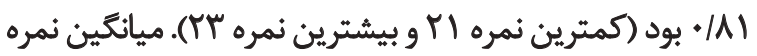

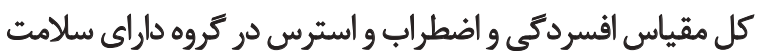

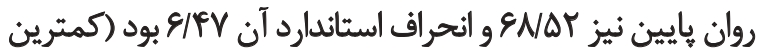

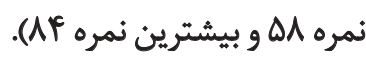

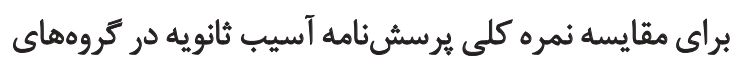

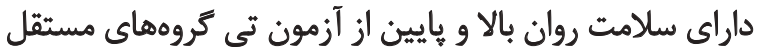

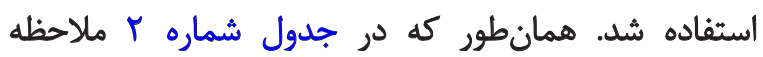

ثانويه در نمونه ايرانى برازش مطلوبي دارد و مى ميوان نتيجه كرفت

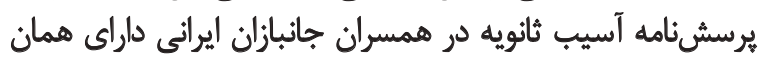
ساختار عاملى است كه در نمونه غيرايراني وجود دارئ دارد.

$$
\text { ووايى همكّرا و افتبراقى }
$$

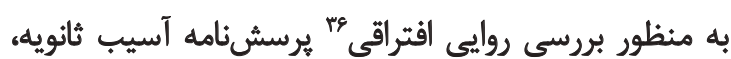

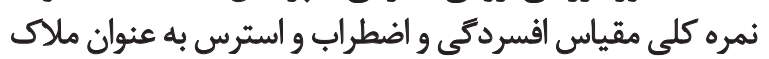

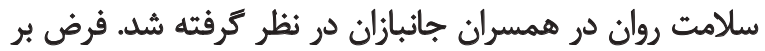

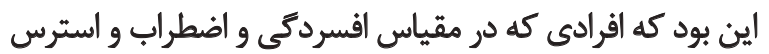

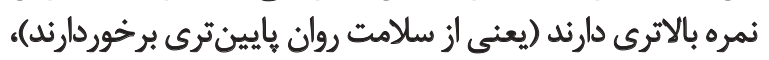

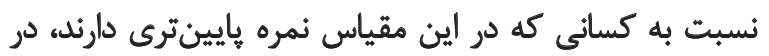

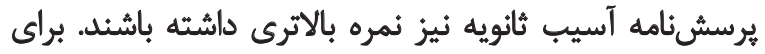

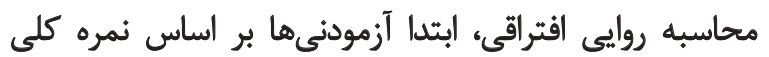
36. Discriminant validity 


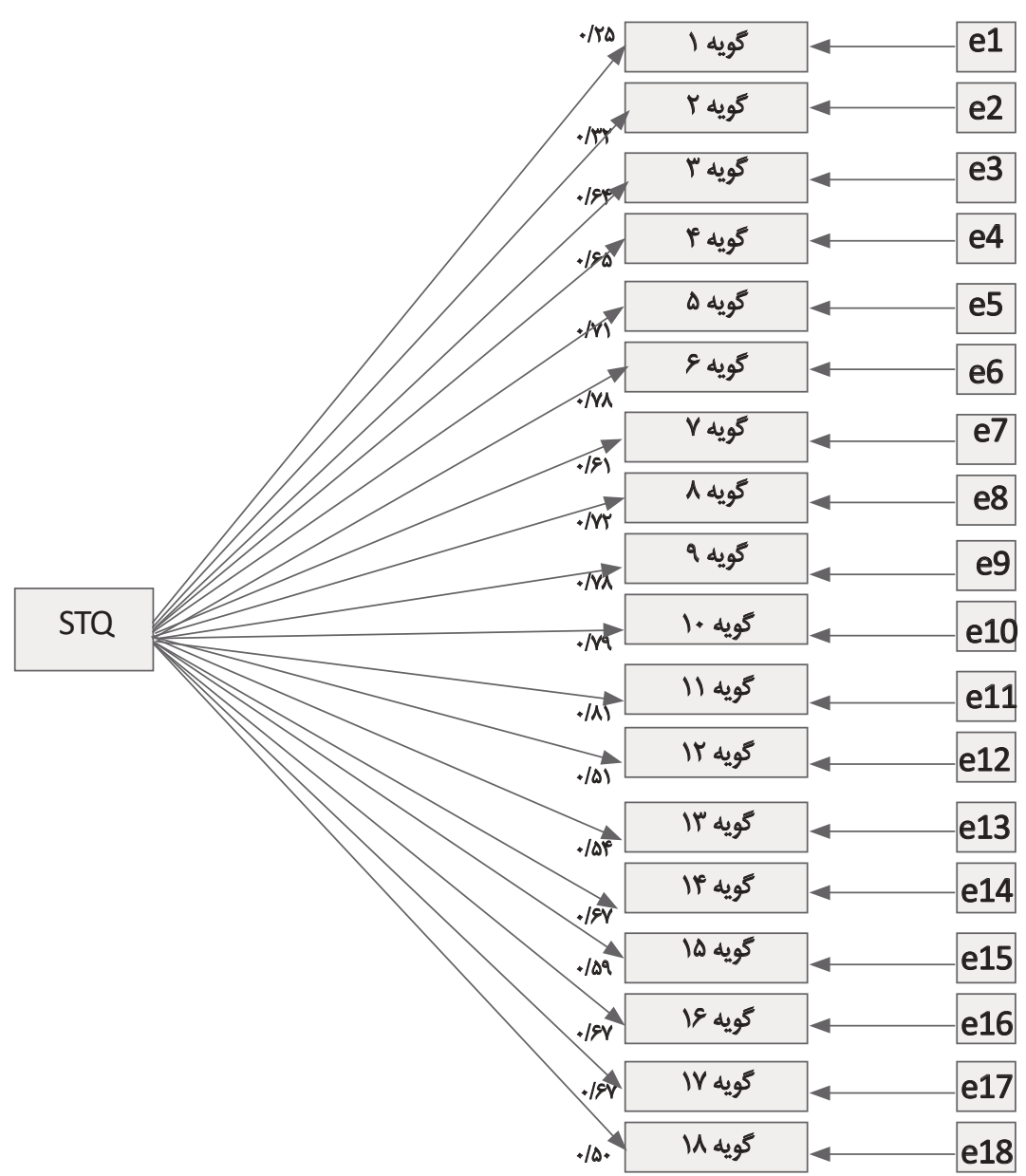

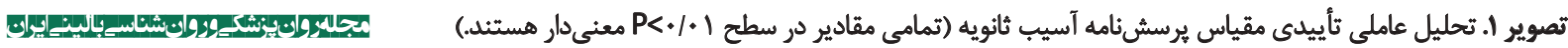

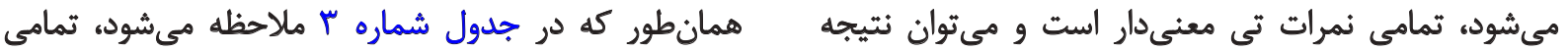

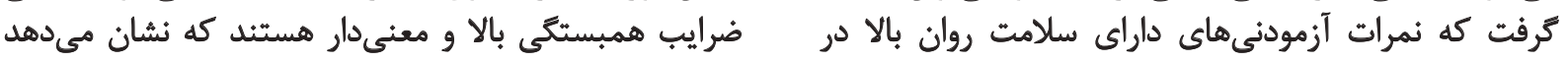

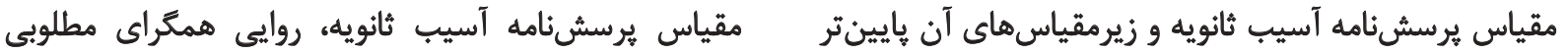

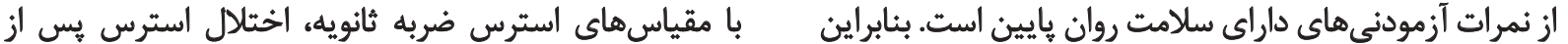

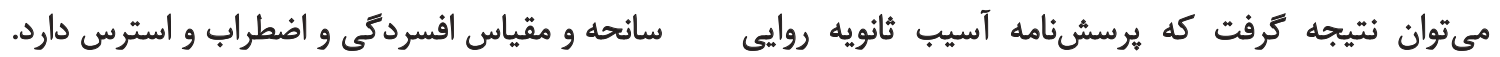

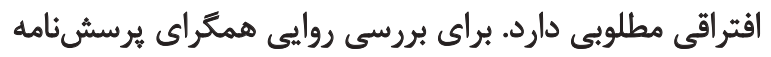

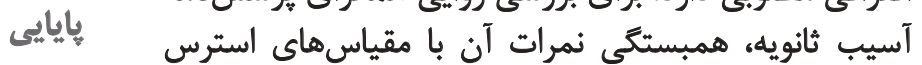

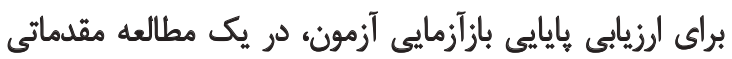

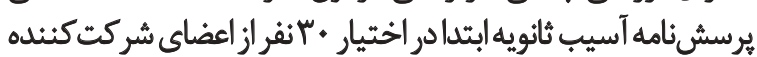

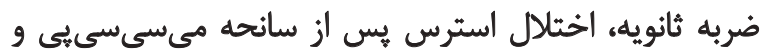

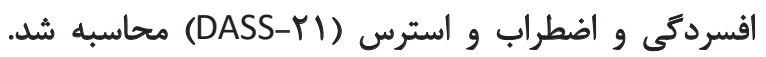

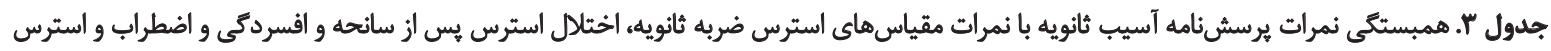

\begin{tabular}{|c|c|c|c|c|c|c|c|c|c|c|}
\hline والضسردئي & أفسردئى & الهيترس & اضطراب & أمتره آلملال & خُودآزازئى & 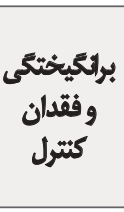 & 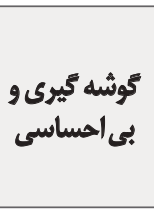 & أز أزجنياب & فيتياس & متغيرها \\
\hline - $M^{e} \Delta^{*}$ & $\cdot N \cdot r^{\prime}$ & $\cdot M I^{*}$ & - Iequ" & $.19 V A^{\circ}$ & $-1 T \Delta A^{*}$ & . 89$)^{\circ}$ & $.17 \cdot 9$ & - Ieme* & - AATA & "يرسشنامي \\
\hline
\end{tabular}


جدول f. ضريب بايايى و همسانى درونى كويهاي مقياس استرس ضربه ثانويه

\begin{tabular}{|c|c|c|c|c|}
\hline حرّ صورت آلفا & با مجمبتوعى & حرف صوّياست & مقر مياسكين & كويه \\
\hline ./9ז8 & . & $r \cdot \Delta / \& M Y$ & $\pi / m$ & سعى مى كنم از افكار و احساساتى كه مشكلات همسرم را به يادم مىآورد اجتناب كنم. \\
\hline.$/ 97 r^{2}$ & 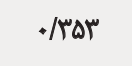 & $r \cdot r / \Lambda \& A$ & $\Pi / \Delta$. & از فعاليتها و موقعيتهايى كه مرا به ياد مشكلات همسرم مى أنازد دورى مى كنهر. \\
\hline.$/ 914$ & .1811 & IqY/AaV & $m / W$ & 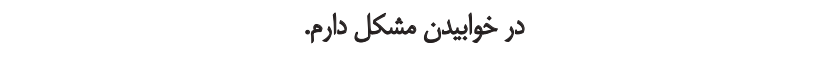 \\
\hline.$/ 91 \wedge$ &.$/ \Delta 94$ & $194 / 8.9$ & $\pi / v \Delta$ & بلدراحتى از جا مىيرم يا وحشتزده مىشوم. \\
\hline.$/ 918$ & $.18 V A$ & $194 / \mathrm{MII}$ & $\operatorname{re} / \mathrm{gV}$ & 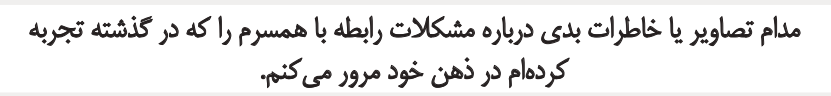 \\
\hline .1914 &.$/ M \Lambda$ & WV/AIA & Me/\&a & كاهى اوقات جيزهايى كه همسرم مى كويد و كارهائى كه انجام مى دهد مرا به وحشت \\
\hline.$/ 919$ & $.10 v 9$ & 199/997 & $r \Delta / r t$ & خوابهاي بدى شبيه حوادثى كه همسرم تجربه كرده است مىبيئه. \\
\hline.$/ 918$ &.$/ 9 N$ & $19 \Delta / . .4$ & $\pi / 9 \Delta$ & افكار ناخواسته و مزاحمى درباره مشكلات همسرم به ذهنم خطور مى كند. \\
\hline .1918 &.$/ M r$ & 19T/RTA & $r \Delta / \bullet 1$ & افكارى كه درباره هشكلات همسرم دارم موقع خواب هزاحمم مى شود و خواب را الز من \\
\hline.$/ 914$ & $\cdot M r$ & $19+/ \Delta F \mid$ & m/1 9 & تصور مى كنم مشكلاتى كه همسرم تجربه كرده ممكن است تأثير منفى در من بكذارد. \\
\hline .1910 &.$M 9$ & $19 . / 215$ & $m / M^{\prime}$ & احساس فشار و تش مى كثم و اين احساس همسرمن است به علت افكارى باشد كه درباره \\
\hline $.194+$ & . /DIT & $198 / 791$ & rq/ar & آرزو دارم روزى بتوانم از مشكلات همسرم دورى كنم. \\
\hline .1919 & $/ \Delta \Delta Y^{\prime}$ & $199 / 7 \mathrm{r}$. & $M / M$ & در بهيادآودن جنبههاى خاص و جزئيات شككلات همسرم ثاثوائم. \\
\hline.$/ 918$ & .1919 & MaVATF & $\Pi / \& \Delta$ & فعاليتهايع راكه موجب لذت من مى شود از دست دادمام. \\
\hline .1911 & /RTA & $|9 P / R T|$ & MQ/AV & مشكلات زيادى در داشتن عواطف كرم و مثبت نسبت به ديكران دارم \\
\hline.$/ 918$ & .1898 & $19 T / \backslash \Delta F$ & me/ar & آينده روشن و خوشبيينانهاى نسبت به آنجه در زندىى كنشتهام بود برايم وجود ندارد. \\
\hline $.191 \mathrm{~V}$ & . IEAT & I91/DFV & $\pi / \Delta 1$ & 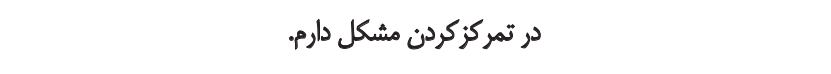 \\
\hline.$/ 941$ & $\cdot / \Delta \cdot r$ & 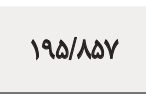 & $\pi / \pi$ & اكر أنجه را كه همسرم تجربه كرده است تجربه كرده بودم، احساس تنش و آسيبيذيرى \\
\hline
\end{tabular}

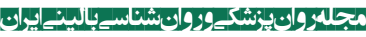

هر يك از كويهها تفاوت جندانى در ضريب آلفاى كرونباخ كل

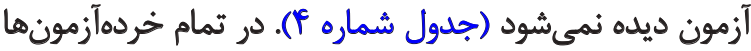

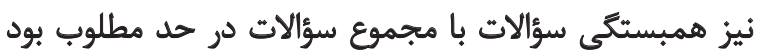

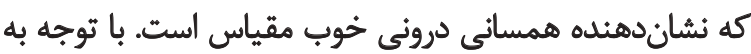

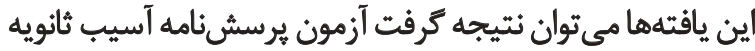

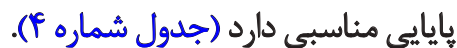
بحث

هدف از يُؤهش حاضر بررسى برخى از ويرُكى هاى روانسنجى
در بثوهش قرار كرفت. يك هفته بعد از اجراى آزمون نيز مجدداً از

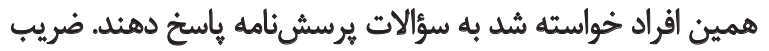

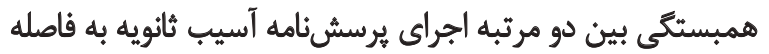

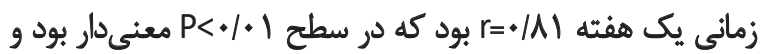
نشان مى داد اين يرسشنامه يايايى بازآزمايى خوبى دارد.

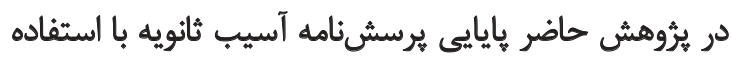

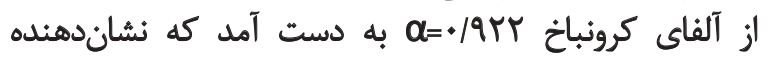

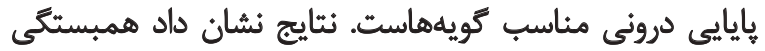

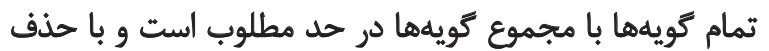


سانحه انجام شد نتّايج نشان داد احساس معنا در زندكى باسطوح

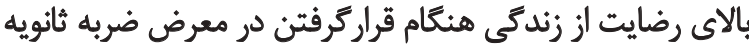

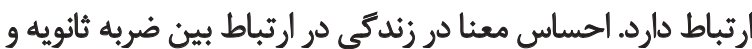

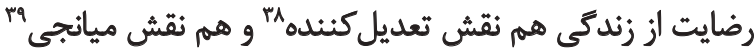

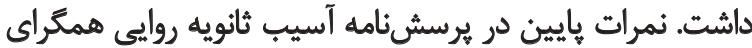

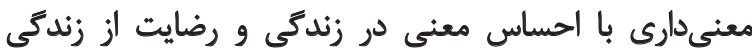

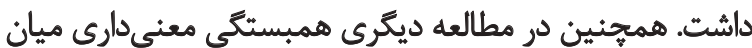

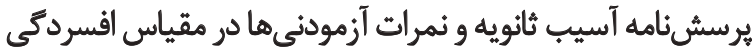

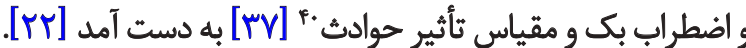

يرسشنامه آسيب ثانويه علاوه بر خانوادههاى سربازان

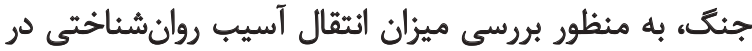

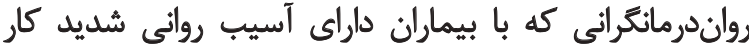

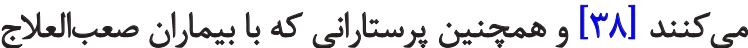

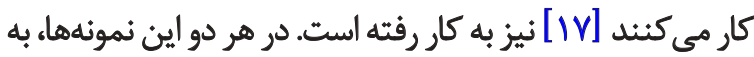

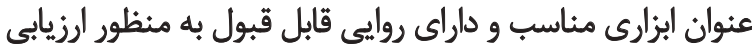

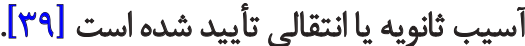

نتايج همبستگى بين دو مرتبه اجراى آزمون در فاصله يك

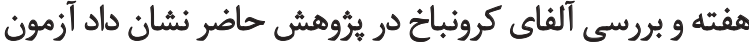

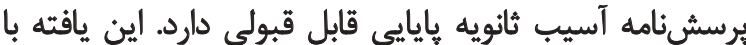

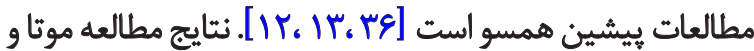

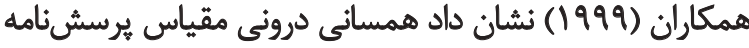

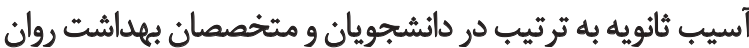

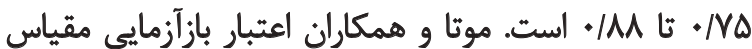

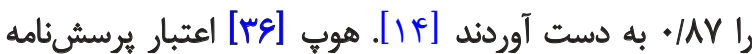

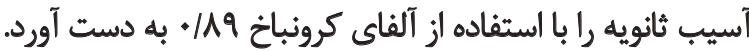

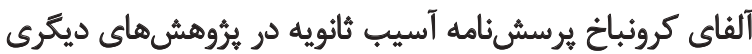

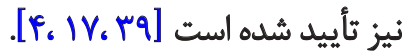

\section{نتيجلمَّيرى}

با توجه به يافتههاي يُروهش حاضر مي ثوان نتيجه كرفت نسخه

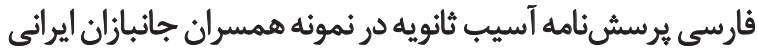

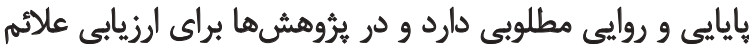

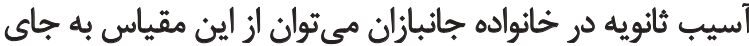

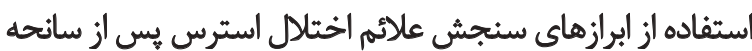

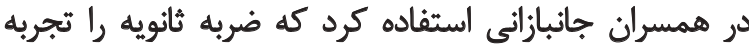

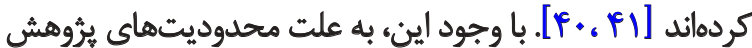

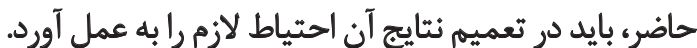
يكى از محدوديتهاى يُروهش حاضر اين بود كه به به علت محدوديتهاى محقق و نياز به حجم نمونه بالائ براى هنجاريابى، بردي،

38. Moderation

39. Mediation

40. Impact of event scale
نسخه فارسى يرسش نامه آسيب ثانويه در همسران جانبازان بودي.

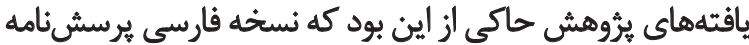

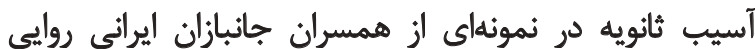

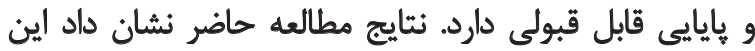

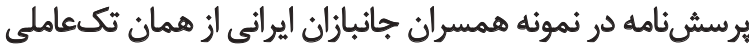

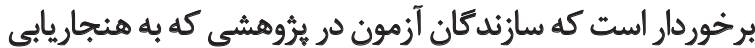

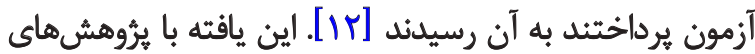

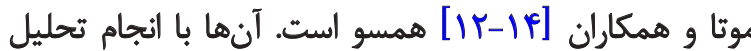

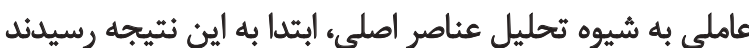

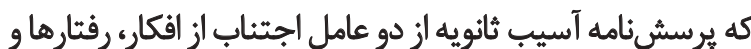
هيجانات و مزاحمت افكار و تصاوير ناخواسته تشكيل شده است.

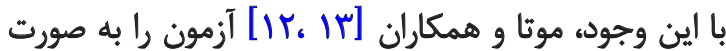

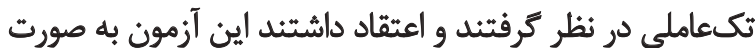

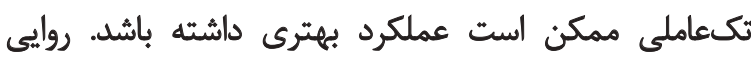

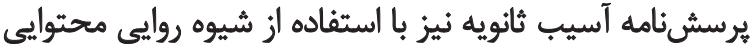

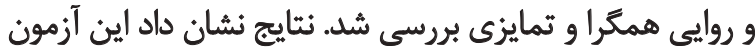

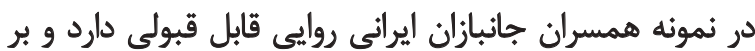

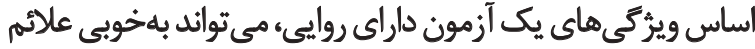

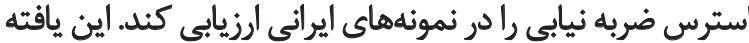

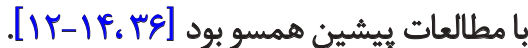

موتا و همكاران [با] براى بررسى روايى واكراى (تمايزى)

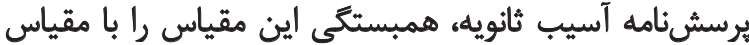

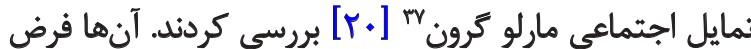

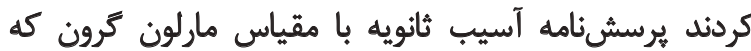

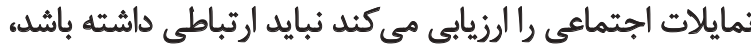

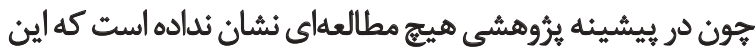

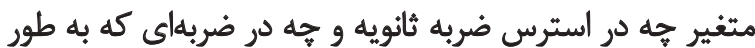

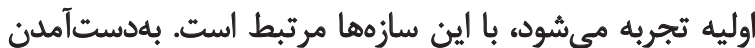

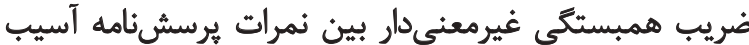

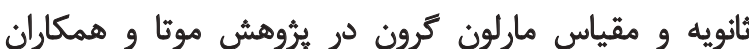

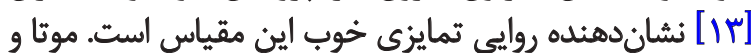

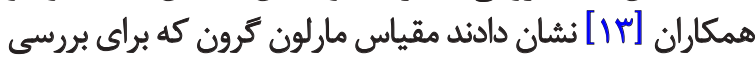

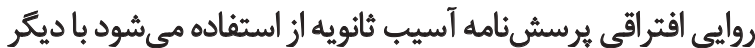

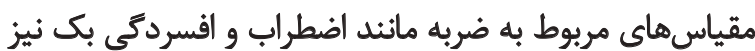

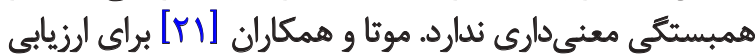

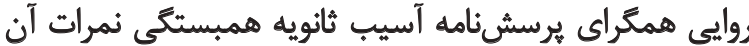

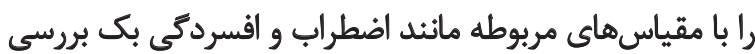

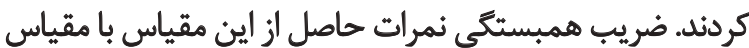

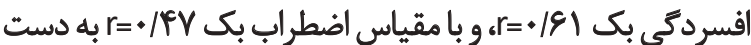

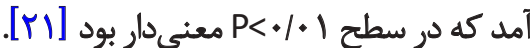
در يُوهشى كه در مراقبان افراد مبتلا به اختلال استرس يس ازي

37. Marlowe-Crowne social desirability scale 
جانبازان اختال استرس يس از سانحه رالز جاثبازان بدون اختلال

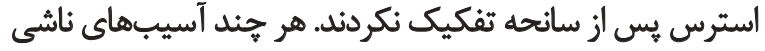

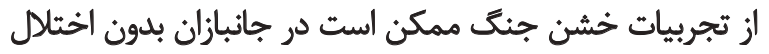

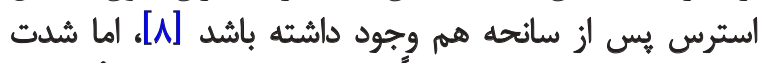

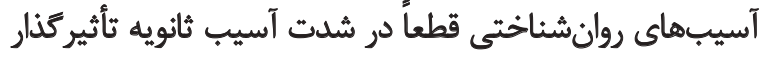

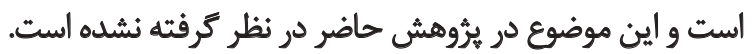

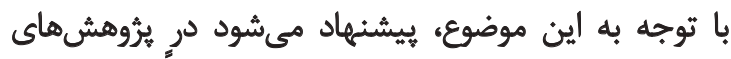

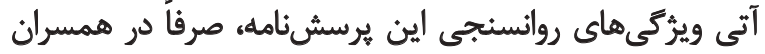

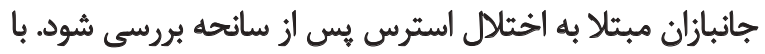

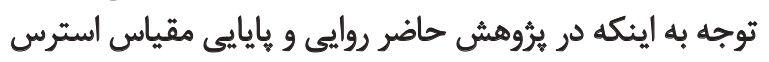

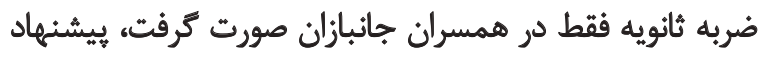

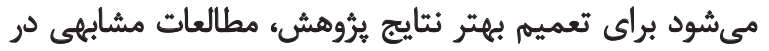

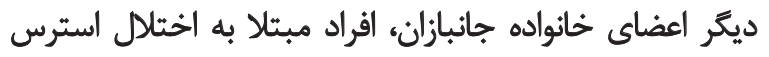

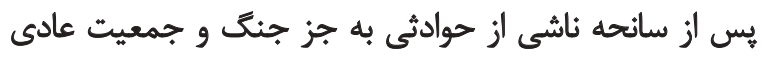

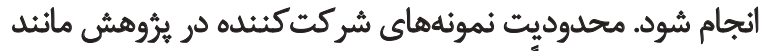

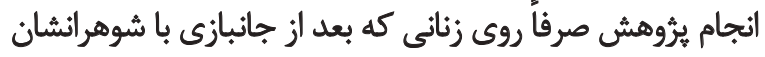

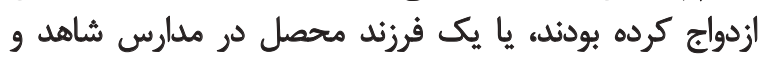

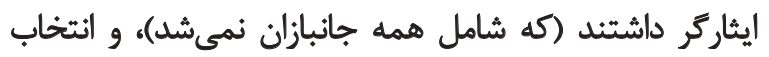

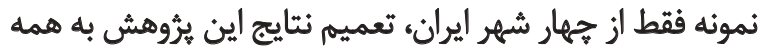

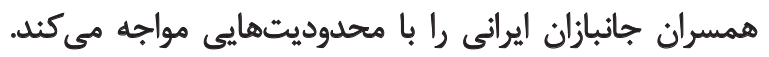

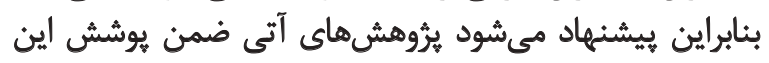

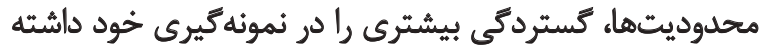

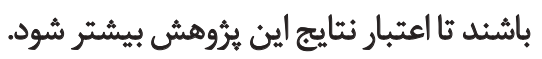

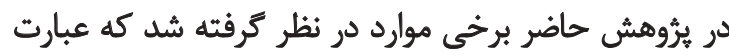

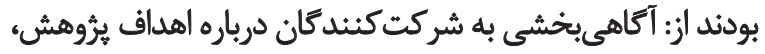

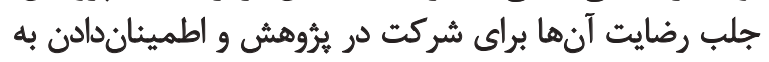

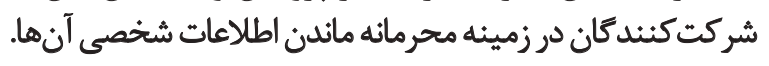
لازم به ذكر است براى بررسى دقيقتر ويزّكى هاى روانسنجى

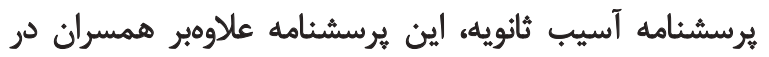

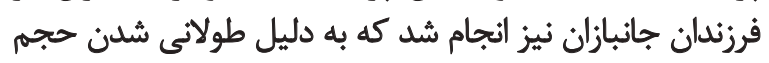

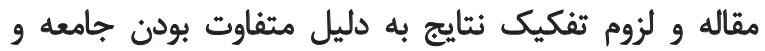

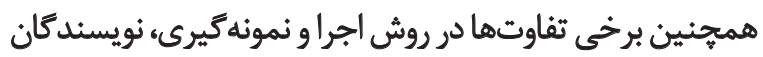

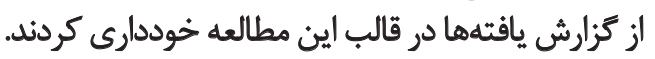

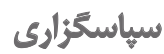

از اساتيد و همجينين تمامى دانشجويان شركت كنينده در

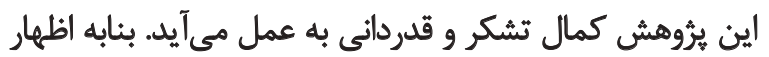

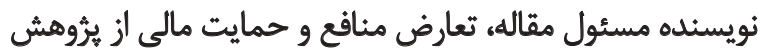

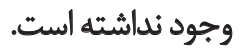




\section{References}

[1] Evans L, Mchugh T, Hopwood M, Watt C. Chronic posttraumatic stress disorder and family functioning of vietnam veterans and their partners. Australian \& New Zealand Journal of Psychiatry. 2003; 37(6):765-72. doi: 10.1080/j.1440-1614.2003.01267.x

[2] Solomon Z. The impact of posttraumatic stress disorder in military situations. Journal of Clinical Psychiatry. 2001; 62(17):11-5.

[3] Figley CR. Burnout as systematic traumatic stress: A model for helping traumatized family members. In: Figley CR, editor. Burnout in families: The systemic costs of caring. New York: CRC Press; 1998.

[4] Dekel R. Posttraumatic distress and growth among wives of prisoners of war: The contribution of husbands' posttraumatic stress disorder and wives' own attachment. American Journal of Orthopsychiatry. 2007; 77(3):419. doi: 10.1037/0002-9432.77.3.419

[5] Dirkzwager AJE, Bramsen I, Adèr H, van der Ploeg HM. Secondary traumatization in partners and parents of dutch peacekeeping soldiers. Journal of Family Psychology. 2005; 19(2):217-26. doi: 10.1037/0893-3200.19.2.217

[6] Figley CR. Compassion fatigue: Psychotherapists' chronic lack of self care. Journal of clinical psychology. 2002; 58(11):1433-41. doi: 10.1002/jclp.10090

[7] Goff BSN, Crow JR, Reisbig AMJ, Hamilton S. The impact of soldiers' deployments to Iraq and Afghanistan: Secondary traumatic stress in female partners. Journal of Couple \& Relationship Therapy. 2009; 8(4):291-305. doi: 10.1080/15332690903246085

[8] Canfield J. Secondary traumatization, burnout, and vicarious traumatization. Smith College Studies in Social Work. 2005; 75(2):81-101. doi: 10.1300/j497v75n02_06

[9] Jenkins SR, Baird S. Secondary traumatic stress and vicarious trauma: A validational study. Journal of Traumatic Stress. 2002; 15(5):423-32. doi: 10.1023/a:1020193526843

[10] Figley CR. Compassion fatigue: Coping with secondary traumatic stress disorder in those who treat the traumatized (Psychosocial stress series). New York: Brunner/Mazel; 1995.

[11] Bride BE, Robinson MM, Yegidis B, Figley CR. Development and validation of the secondary traumatic stress scale. Research on Social Work Practice. 2004; 14(1):27-35. doi: $10.1177 / 1049731503254106$

[12] Motta RW, Joseph JM, Rose RD, Suozzi JM, Leiderman LJ. Secondary trauma: Assessing inter generational transmission of war experiences with a modified Stroop procedure. Journal of Clinical Psychology. 1997; 53(8):895-903. doi: 10.1002/(sici)10974679(199712)53:8<895::aid-jclp14>3.0.co; 2-f

[13] Motta R. Discriminant validation of the modified secondary trauma questionnaire. Journal of Psychotherapy in Independent Practice. 2002; 2(4):17-24. doi: 10.1300/j288v02n04_02

[14] Motta RW, Kefer JM, Hertz MD, Hafeez S. Initial evaluation of the secondary trauma questionnaire. Psychological Reports. 1999; 85(3):997-1002. doi: 10.2466/pr0.1999.85.3.997

[15] Lombardo KL, Motta RW. Secondary trauma in children of parents with mental illness. Traumatology. 2008; 14(3):57-67. doi: $10.1177 / 1534765608320331$
[16] Motta RW. Secondary trauma in children and school personnel. Journal of Applied School Psychology. 2012; 28(3):256-69. doi: 10.1080/15377903.2012.695767

[17] Zimering R, Munroe J, Gulliver SB. Secondary traumatization in mental health care providers. Psychiatric Times. 2003; 20(4):24-36.

[18] Renshaw KD, Allen ES, Rhoades GK, Blais RK, Markman HJ Stanley SM. Distress in spouses of service members with symptoms of combat-related PTSD: Secondary traumatic stress or general psychological distress. Journal of Family Psychology. 2011; 25(4):461-9. doi: 10.1037/a0023994

[19] Human HA. [Educational and psychological measurement and technique of developing the test (Persian)]. Tehran: Selseleh; 2001

[20] Crowne DP, Marlowe D. A new scale of social desirability independent of psychopathology. Journal of Consulting Psychology. 1960; 24(4):349-54. Doi: 10.1037/h0047358

[21] Motta RW, Newman CL, Lombardo KL, Silverman MA. Objective assessment of secondary trauma. International Journal of Emergency Mental Health. 2004; 6(2):67-74. PMID: 15298077

[22] Gawrych AL. PTSD in firefighters and secondary trauma in their wives. New York: Hofstra University; 2010

[23] Ahmadi K, Rezapour Y, Davoudi F, Saberi M. [Investigate of validity and reliability of secondary trauma stress scale for evaluation of PTSD symptoms in samples of warfare victims' wives (Persian)]. Iranian Journal of War and Public Health. 2013; 5(3):4757.

[24] Rezapour Mirsaleh Y, Ahmadi K, Davoudi F, Mousavi SZ. [Validity, reliability, and factor structure of secondary trauma stress scale (stss) in a sample of warfare victims' children (Persian)] Iranian Journal of Psychiatry and Clinical Psychology. 2014 20(2):134-43.

[25] Lovibond PF, Lovibond SH. The structure of negative emotional states: Comparison of the depression anxiety stress scales (DASS) with the Beck depression and anxiety inventories. Behaviour Research and Therapy. 1995; 33(3):335-43. doi: 10.1016/00057967(94)00075-u

[26] Sahebi A, Asghari MJ, Salari RS. [Validation of depression anxiety and stress scale (DASS-21) for an Iranian population (Persian)]. Iranian Psychologists. 2005; 4(1):299-313.

[27] Vreven DL, Gudanowski DM, King LA, King DW. The civilian version of the Mississippi PTSD scale: A psychometric evaluation. Journal of Traumatic Stress. 1995; 8(1):91-109. doi: 10.1002/ jts. 2490080107

[28] Gudarzi MA. [Reliability and validity of Mississippi PTSD Scale (Persian)]. Journal of Psychology. 2003; 7(2):153-78

[29] Broder HL, McGrath C, Cisneros GJ. Questionnaire development: face validity and item impact testing of the child oral health impact profile. Community Dentistry and Oral Epidemiology. 2007; 35(s1):8-19. doi: 10.1111/j.1600-0528.2007.00401.x

[30] Yaghmale F. Content validity and its estimation. Journa of Medical Education. 2009; 3(1):25-27. doi: 10.22037/jme. v3i1.870

[31] Waltz CF, Strickland OL, Lenz ER. Measurement in nursing and health research. New York: Springer Publishing Company; 2010 
[32] Polit DF, Beck CT. The content validity index: Are you sure you know what's being reported? Critique and recommendations. Research in Nursing \& Health. 2006; 29(5):489-97. doi: 10.1002/ nur.20147

[33] Polit DF, Beck CT, Owen SV. Is the CVI an acceptable indicator of content validity? Appraisal and recommendations. Research in Nursing \& Health. 2007; 30(4):459-67. doi: 10.1002/nur.20199

[34] Lawshe CH. A qualitative approach to content validity. Personnel Psychology. 1975; 28(4):563-75. doi: 10.1111/j.1744-6570.1975. tb01393.x

[35] Ghasemi V. [Structural modeling in social science research (Persian)]. Tehran: Jame'e Shenasan Pub; 2010.

[36] Hope NL. When caring hurts: The significance of personal meaning for wellbeing in the presence of secondary traumatic stress [Master thesis]. British Columbia: Trinity Western University; 2006.

[37] Horowitz M, Wilner N, Alvarez W. Impact of event scale: A measure of subjective stress. Psychosomatic Medicine. 1979; 41(3):209-18. doi: 10.1097/00006842-197905000-00004

[38] Sabin Farrell R, Turpin G. Vicarious traumatization: Implications for the mental health of health workers. Clinical Psychology Review. 2003; 23(3):449-80. doi: 10.1016/s0272-7358(03)00030-8

[39] Suozzi JM, Motta RW. The relationship between combat exposure and the transfer of trauma like symptoms to offspring of veterans. Traumatology. 2004; 10(1):17-37. doi: 10.1528/ trau.10.1.17.31151

[40] Ahmadi KB, Reshadatjoo M, Karami GR, Anisi J. [Vicarious PTSD in Sardasht chemical warfare victims' wives (Persian)]. Journal of Behavioral Sciences. 2009; 3(3):195-199.

[41] Kh A, Reshadatjoo M, Anisi J. [Evaluation of secondary post traumatic stress disorder in chemical warfare victims' children (Persian)]. Journal of Military Medicine. 2010; 12(3):153-9. 